\title{
POSITION PAPER
}

\section{Diagnosis and treatment of Paget's disease of bone}

\author{
Robert G. Josse, MB ${ }^{1}$ \\ David A. Hanley, MD² \\ David Kendler, MD $^{3}$ \\ Louis-Georges Ste Marie, $\mathrm{MD}^{4}$ \\ Jonathan D. Adachi, MD 5 \\ Jacques Brown, MD6
}

${ }^{1}$ Division of Endocrinology and Metabolism, Department of Medicine, St. Michael's Hospital, University of Toronto

${ }^{2}$ Division of Endocrinology and Metabolism, Department of Medicine, University of Calgary Health Sciences Centre

${ }^{3}$ Division of Endocrinology and Metabolism, Department of Medicine, University of British Columbia ${ }^{4}$ Division of Endocrinology and Metabolism, Department of Medicine, CHUM Research Centre, Université de Montréal

${ }^{5}$ Division of Rheumatology, Department of Medicine, St. Joseph's Healthcare - McMaster University ${ }^{6}$ Division of Rheumatology, Department of Medicine, Le Centre hospitalier universitaire de Québec (CHUQM), Laval University

Manuscript submitted 24th April, 2007

Manuscript accepted 11th May, 2007

Clin Invest Med 2007; 30 (5): E210-E223.

\section{Abstract}

Paget's disease of bone (PDB) is a metabolic bone disease characterized by increased bone resorption followed by excessive unregulated bone formation. This results in weakened, deformed bones of increased mass in which the collagen fibres assume a haphazard irregular mosaic pattern instead of the normal parallel symmetry. PDB rarely occurs before middle age and its prevalence increases steadily with age. The overall prevalence in Caucasians is approximately 3\%; although it appears to be declining. There is a geographic variation in prevalence, 40 with highest rates found in the UK. PDB affects both men 41 and women, with a slight predominance in men. PDB may 42 be asymptomatic or symptomatic, depending on the bones 43 involved; the most common symptom is pain in the af44 fected bone. While its aetiology remains elusive, genetic 45 factors and environmental influences are implicated. In
2002, guidelines for PDB management were developed in Great Britain and have gained worldwide acceptance. In this position paper, an Expert Panel of Canadian endocrinologists and rheumatologists examines current evidence on the diagnosis and treatment of PDB to provide Canadian recommendations. In general, diagnosis may be confirmed both by X-ray and by the biochemical marker serum alkaline phosphatase, which is elevated in $85 \%$ of individuals with untreated active PDB. Treatment is indicated for all patients with symptoms and for asymptomatic patients with active PDB in areas of the skeleton with the potential to produce complications of clinical importance. The Panel recommends treating PDB with bisphosphonates that have demonstrated superior efficacy and remission rates. 
1 Paget's disease of bone (PDB) is a condition of unknown aetiology that involves accelerated bone resorption followed by the deposition of dense and disorganized bone matrix. It normally affects the elderly. Often asymptomatic, the patient may present with symptoms depending on the bones involved, the most common being pain in the affected bone. Neurological, hearing, vision, cardiac, arthritic and oncological complications as well as bone deformities have been 10 described. Although its aetiology remains elusive, considerable advances have been made in understand2 ing its pathology and in treating the condition. In 3 2002, guidelines for the management of PDB were 4 developed by the Bone and Tooth Society of Great 5 Britain (now Bone Research Society) in association 6 with the National Association for the Relief of Paget's 7 Disease. ${ }^{1}$ These guidelines have gained worldwide 18 acceptance. In this position paper, endorsed by the 9 Canadian Society of Endocrinology and Metabolism 20 (CSEM), an Expert Panel has examined current evidence on the diagnosis and treatment of PDB in an effort to provide Canadian recommendations.

\section{Epidemiology}

Radiological and autopsy prevalence studies indicate that PDB rarely occurs before middle age and its prevalence increases steadily with age. ${ }^{1}$ A large-scale study from the United Kingdom conducted in 1994 showed that while the disorder was present in $0.3 \%$ of both men and women aged 55 to $59 \mathrm{yr}$, the prevalence increased to $6.9 \%$ of men and $5.8 \%$ of women aged $\geq 85$ yr. $^{2}$ However, it now appears that the overall prevalence of the disease is declining. This same British study compared its findings with an identical study conducted in 1974 and found that the prevalence of PDB in 1994 was only $40 \%$ of that observed $20 \mathrm{yr}$ earlier (men: $7 \%$ vs. $3.8 \%$; women $2.5 \%$ vs. $1.6 \%$ ). Corroborating data from New Zealand show that not only is Paget's Disease occurring at a later age but the presentation is less severe than previously. The authors suggest that this may mean that environmental influences, which may be waning, (on a background of genetic predisposition) may be important in the etiology of Paget's disease. ${ }^{3}$ The same UK authors have reported an update on the epidemiology of Paget's disease of bone since their 1999 publication. Over the period 1988-1999, the incidence rate of clinically diagnosed Paget's disease was found to be 5 per 10,000 person years among men and 3 per 10,000 person years among women $>75 \mathrm{yr}^{2}$

There is a distinct geographical variation in the prevalence of the disease. Epidemiological studies have demonstrated high rates of PDB in the UK (even within localized areas of England), with somewhat lower rates in Australia, North America, and Western Europe. PDB is uncommon among African Blacks, Asians, Scandinavians, Irish, Swiss, and Southern Europeans. ${ }^{4}$ PDB affects both men and women, with a slight predominance in men. ${ }^{7}$

\section{Aetiology}

Whereas several aetiological factors have been considered, most have not been positively associated with PDB. The striking geographic variation in disease prevalence, as well as the fact that its incidence around the world is apparently declining, ${ }^{1,3,8}$ may be explained in part by environmental influences, though none in particular appears to have been confirmed. Changes in calcium intake during childhood have been postulated since case-control studies have shown an association between low dietary calcium and PDB. ${ }^{8}$ Studies over the last two or three decades have also suggested that paramyxoviruses may play a role in the aetiology of the disease; measles, respiratory syncytial, and canine distemper viruses have all been implicated. ${ }^{6}$ Most of the studies undertaken to investigate a viral aetiology have demonstrated the presence of one or more paramyxoviruses in pagetic bone or bone marrow cells. ${ }^{9}$ Recently, it has been demonstrated that Canine Distemper Virus (CDV) can infect and replicate in human osteoclast precursors. ${ }^{6}$ The mechanism of action for the osteoclastogenesis may involve activation of sequestosome 1/P62 and NF- $\mathrm{KB}$ activation. This process also involves ubiquitin. ${ }^{8} \mathrm{Ul}-$ trastructural studies of the bone from patients with PDB have also suggested the possible causality of a slow virus infection analogous to subacute sclerosing 
panencephalitis, a childhood condition that follows classical measles virus infection. ${ }^{9}$

There is also compelling evidence to support the contribution of genetic factors in the aetiology of PDB. Current data suggest that mutations in the sequestosome 1/p62 gene (SQSTM1/P62) conferred disease susceptibility but may not be causative, with some environmental influence being required to allow expression of the disease. ${ }^{3}$ In fact, a unifying hypothesis suggests that PDB may arise as the result of a genetic predisposition to a viral infection. ${ }^{8}$

12

\section{Genetics}

Familial clustering of PDB has been documented frequently and, in families, the disorder appears to be transmitted by an autosomal dominant mode of inheritance with incomplete penetrance. ${ }^{10,11}$ The suggestion of a genetic linkage with the classical, adult-onset PDB was first obtained with the HLA locus on chromosome 6.12,13 Since 1997, six other loci have been reported: PDB2 on chromosome 18,14 PDB3 and PDB4, both on chromosome 5q, ${ }^{15}$ PDB5 and PDB6 on chromosomes 2 and 10, respectively, ${ }^{16}$ and PDB7 on chromosome $18 .{ }^{17}$ Of these loci, only the PDB3 locus has been confirmed in an independent sample, ${ }^{16}$ and the underlying gene identified as the SQSTM1/P62.18 The first reported mutation in this gene results in a change from the amino acid proline to a leucine at position 392 of the protein sequence (P392L). The P392L mutation was associated with two different haplotypes in a Quebec sample, suggesting that it was a recurrent mutation, i.e., coming from two different ancestral mutation events. ${ }^{18}$ The P392L mutation was the only mutation found in unrelated PDB cases and families from Quebec. This mutation was originally observed in 18 out of $112(16 \%)$ unrelated individuals and in $11(46 \%)$ of the 24 families. Updated information from the same authors show that overall $36.8 \%$ and $8.3 \%$ of the familial and unrelated cases studied harboured one of the reported mutations in SQSTM1/ P62. The P392L mutation accounted for $64.1 \%$ of familial and $85.5 \%$ of unrelated carriers of any of the SQSTM1/P62 mutations. These data again highlight the potentially important role played by this gene in
Paget's disease susceptibility. ${ }^{19}$ Almost all SQSTM1 mutations reported to date cluster in the ubiquitin associating domain UBA. These are loss-of-function mutations and suggest that loss of the interaction with ubiquitin is probably critical in the pathogenesis of the disease. ${ }^{20}$

\section{Clinical history and manifestations}

Although PDB is often asymptomatic, $5 \%$ of patients experience symptoms, ${ }^{21}$ including: $:, 7$

- bone and joint pain (occurring in the affected bone)

- joint pain from osteoarthritis in joints contiguous to pagetic bones

- bone deformities that may result in the bowing of a limb or increased skull size

- fracture, since pagetic bone is weaker than normal bone

- hearing loss due to temporal bone involvement and $8^{\text {th }}$ nerve damage

- headache, when the skull is affected by PDB

- hypercalciuria and hypercalcemia due to accelerated bone resorption induced by immobilization

- neurological complications (radicular paresis or paraparesis) due to vascular steal phenomenon, and/ or

- nerve entrapment syndromes. (e.g., nerve root compression caused by the impingement on nerve roots (mainly cranial nerves or spinal nerves) as they exit foramina narrowed by Pagetic bony involvement.)

The primary pathological abnormality in patients with PDB is increased bone resorption, followed by abundant formation of new bone that is disorganized and of poor quality. ${ }^{5}$ Pagetic lesions may be found in only one bone (monostotic form; 17\%) - predominantly the pelvis, spine, skull or long bones like the tibia - or in multiple bones (polyostotic form), which is generally more symptomatic.5,7,22 Interestingly, PDB usually remains localized to the bones identified at first diagnosis and patients rarely develop new lesions in previously unaffected bones during the course of the disease. ${ }^{5,7}$ Instead, lesions tend to increase in size until treated. 5 The most serious complication of 


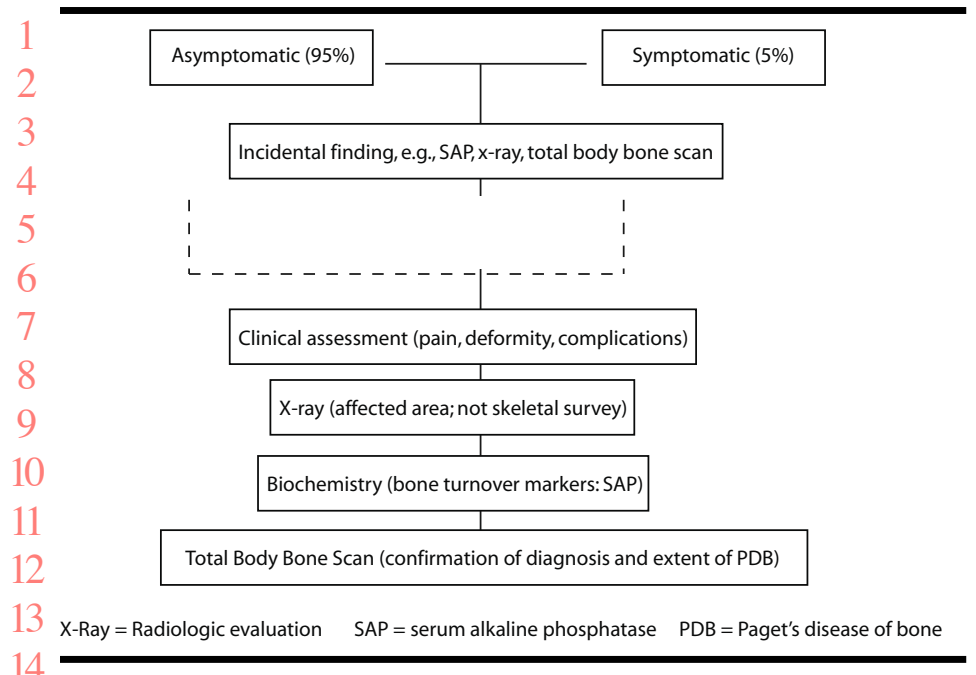

FIGURE 1. Diagnosis of Paget's Disease of Bone

16

$17 \mathrm{PDB}$ is osteosarcoma, although it is quite rare, occur8 ring in less than $1 \%$ of patients. ${ }^{7}$

19

\section{Burden of illness}

Given that most patients with PDB are asymptomatic and, therefore, may be undiagnosed, it is difficult to assess the true burden of PDB in the general population. There appears to be no reliable information available regarding the treatment and associated costs of PDB. ${ }^{1}$ Calculating the cost of treatment is more complex than simply multiplying the acquisition cost of the drugs by the duration of treatment since factors such as re-treatment, complications of the disease that have been averted, and adverse events as a result of treatment must be factored into the equation. ${ }^{23}$

\section{Diagnosis}

PDB may present with obvious signs or symptoms or it may be an incidental finding during the investigation of other conditions ${ }^{1}$ (see Figure 1) such as the signs and symptoms listed above.

\section{Radiological/Bone scintigraphy}

41 Plain radiographs: ${ }^{1}$ The diagnosis of PDB is primarily 42 radiological. PDB has characteristic features on X-ray which usually do not leave the diagnosis in doubt, but the differential diagnosis may include metastatic disease, osteomyelitis and primary bone tumours. Where plain radiology is equivocal, computed tomography may be considered. The recommendation is that the diagnosis of PDB be confirmed with plain radiology of at least one skeletal site in all symptomatic patients with the condition. Plain radiographs are also valuable in the diagnosis of secondary complications of PDB (e.g., arthritis or fracture).

Whereas full skeletal X-ray surveys are not usually necessary to determine the extent of skeletal involvement, a baseline bone scan is important since it provides an indication of the extent of the disease. Isotopic bone scans (scintigraphy $)^{1}$ are more sensitive than $\mathrm{x}$-rays in highlighting areas affected by PDB. They are recommended (where available) for patients with asymptomatic or symptomatic PDB to assess the extent of skeletal involvement. Targeted radiographs at the sites with increased uptake on the bone scintigraphy should be done to assess the nature of PDB involvement at these sites. A follow-up bone scintigraphy done six months after treatment, showing normalized uptake, may be useful in assessing therapeutic response in monostotic disease with baseline SAP within the normal range. Scintigraphy may also be considered if the clinician wishes to determine whether the disease is in remission; i.e., total suppression of bone activity, as evidenced by normal radionuclide uptake..

\section{Biochemical}

Since PDB is associated with increased bone turnover, increased activity of markers of bone turnover is to be expected in active disease. Total alkaline phosphatase (ALP, or SAP for serum alkaline phosphatase) is elevated in $85 \%$ of patients with untreated PDB.1,23 There is a strong relationship between the extent of disease activity measured by scintigraphy and the degree of the elevation of SAP in untreated PDB. 1 "Normal" SAP within the context of PDB may be indicative of monostotic disease and can be encountered in primary lytic forms of PDB. Comparisons between bone specific ALP and total SAP have demonstrated that the former is the more sensitive of the two., ${ }^{1,23}$ However, since bone specific ALP is less readily available and 
TABLE 1. Decision to Treat

Treatment not indicated

Asymptomatic patients

- Normal SAP, normal bone scan, x-ray abnormality consistent with PDB

Pharmacotherapy indicated (patient definitively diagnosed with PDB)

SAP elevations

- Asymptomatic: typically $1.5 \times$ ULN, although any level of SAP in a site with potential complication

- Symptomatic: any level of SAP

Bone scan and $\mathrm{x}$-ray findings diagnostic of PDB regardless of SAP

Specific situations:

1. Pain

2. Fracture or fissure of pagetic bone

3. Osteolytic fronts in pagetic bone

4. Planned orthopedic surgery around pagetic bone

5. Neurological compromise or risk: deafness, spinal nerve compromise

6. Pagetic lesions close to weight-bearing joint e.g. acetabulum, knee

7. Hypercalcemia due to PDB (consider other or additional diagnosis)

8. High cardiac output failure (a rare occurrence)

$\mathrm{SAP}=$ serum alkaline phosphatase

$\mathrm{X}$-Ray $=$ radiologic evaluation

$\mathrm{ULN}=$ upper limit of normal

PDB $=$ Paget's disease of bone

does not exhibit major advantages over total SAP, the latter is recommended as the standard marker of bone turnover in patients with PDB, reserving the use of bone specific ALP as a marker for those PDB patients without elevated total SAP. 1,23

A variety of biochemical tests that reflect bone matrix resorption (e.g., urinary and serum deoxypyridinoline, N-telopeptide [NTX], and alpha- and beta-Ctelopeptides) also provide good indices of disease activity and an immediate index of response to therapy. But despite increasing evidence that these markers of bone resorption may be even more sensitive markers, ${ }^{24,25}$ these tests are not as universally available as SAP. Additionally, the controlled clinical trials in PDB have used SAP for both the entry criteria and as the primary outcome.

\section{Histological}

Bone biopsy is rarely required in diagnosing PDB but may be useful in differentiating it from osteoblastic metastases or osteosarcoma, ${ }^{1}$ particularly when imaging studies such as plain radiographic assessments complemented by CT scan $( \pm$ MRI) are equivocal. Ideally, the bone biopsy should be done after double tetracycline labelling, with the bone sample being processed without decalcification.

Histology usually demonstrates an increase in 37 bone resorption with large multinucleated ("giant") osteoclasts with increased bone marrow fibrosis surrounding bone trabeculae. In addition, there is often accelerated disorganized bone formation with increases seen in osteoid bone surfaces, osteoid bone volume, and bone mineralization rate (therefore excluding osteomalacia). This high bone formation rate leads to an abnormal "woven" bone pattern instead of 45 
the normal lamellar bone texture. The increased bone volume could also lead to a loss of distinction between cortical bone and trabecular bone. Despite this osteosclerosis, because of altered bone quality, pagetic bone is more fragile and prone to fracture. Vertebral compression fractures account for an appreciable proportion of the fractures seen in PDB, and are often at uninvolved sites. ${ }^{26}$ Finally, the bone is often hypervascular, which leads to increased bleeding during orthopaedic surgery undertaken without prior antiresorptive treatment.

12

\section{Indications for therapy}

Numerous criteria exist for the decision to treat PDB (see Table 1). Bone pain is the only symptom of PDB for which there is firm evidence that antipagetic therapy confers clinical benefit, thus pain in pagetic bone is a definite indication for antipagetic therapy. ${ }^{1}$ However, one must distinguish bone pain due to pagetic activity (i.e., "metabolic pain") from pain due to bone and/or joint deformity as a consequence of the disease (i.e., "arthritic pain"). While the former is usually present at rest, the latter is present during mobilization of the joint and may therefore respond to NSAIDs and/or other analgesics, but not to antipagetic drug therapy.

Fracture is a fairly common complication of PDB but, the effect of antipagetic therapy on fracture rates has not been adequately studied. Treatment of PDB with the goal of reducing fracture risk or to improve fracture healing has not been demonstrated, although it may be considered. ${ }^{1}$

The effects of antipagetic therapy on bone deformity are similarly unclear. ${ }^{1}$ While therapy may be warranted in managing facial deformities due to $\mathrm{PDB},{ }^{1}$ whether or not it is effective solely for the prevention of deformity elsewhere remains to be seen. However, it is generally believed that initiation of therapy halts the progression of the disease.

There are no specific recommendations about treatment of osteolytic lesions associated with PDB in the absence of other indications for treatment. ${ }^{1}$ However, since osteolytic lesions in lower limbs are a sig- nificant cause of bone fragility, ${ }^{22}$ and there is evidence suggesting that all bisphosphonates except etidronate promote radiological healing of osteolytic lesions in $\mathrm{PDB},{ }^{1}$ treatment with a bisphosphonate may be indicated. ${ }^{21}$

Although PDB increases the risk of osteoarthritis, ${ }^{1}$ there is limited evidence ${ }^{26}$ that antipagetic therapy affects its development or progression. In a 12-year follow-up of 41 patients with PDB, osteoarthritic complications occurred in $62 \%$ of the patients who reduced their SAP by $50 \%$ after treatment, compared with $33 \%$ of those who normalized their SAP after treatment. ${ }^{27}$ Thus, it is reasonable to treat patients in whom there is uncertainty as to the cause of pain because there is often difficulty distinguishing between pain that emanates from PDB or pain due to osteoarthritis in an adjacent joint. ${ }^{1}$ When PDB is asymptomatic, but involves bone adjacent to a major weightbearing joint such as hip or knee, many experts would also favour treatment to avoid expansion of PDB into the joint.

Deafness is a fairly common complication of PDB but the effect of antipagetic therapy vis-à-vis hearing loss is equivocal. Nevertheless, due to the irreversibility of hearing loss, it is recommend that patients with PDB of the skull base be treated to minimize the risk of progression and later deafness. ${ }^{1}$

Although spinal cord lesions are a relatively rare complication of PDB, bisphosphonates have been shown to improve neurological function in patients with this complication. ${ }^{1}$ However, since the condition usually occurs as a result of a vascular steal phenomenon related to hypervascular pagetic bone of an affected vertebra, it is recommended that patients who develop neurological symptoms as a result of spinal PDB be treated medically. Surgical decompression should be reserved for patients unresponsive to medical therapy. ${ }^{1}$ Note that prior medical therapy is necessary to reduce the risk of bleeding during surgery performed on a bone with active PDB.

Another rare complication of PDB, hypercalcemia may result from a combination of increased bone turnover and immobilization, but is almost always due to the presence of another cause of hypercalcemia 4 such as primary hyperparathyroidism. Clinical obser- 
vations indicate that, given their efficacy in treating hypercalcemia due to most other causes, bisphosphonates may also be helpful in these circumstances. ${ }^{1}$

\section{Treatments for PDB}

\section{Symptomatic}

The main symptom of PDB is pain; although only $5 \%$ 9 of patients actually experience it. ${ }^{21}$ Patients with pain must be carefully assessed to determine its likely cause. Pain arising from elevated bone turnover (e.g., as indicated by elevated SAP) responds well to osteoclast inhibitors (e.g., bisphosphonates), whereas pain from nerve compression (which may arise from bone deformity or coexisting arthritis) does not and should be treated with standard pain relievers (e.g., analgesics, NSAIDs, etc.). Some patients also benefit from combination therapy comprising an analgesic and a low-dose tricyclic antidepressant. Physical methods of pain control including acupuncture, transcutaneous electrical nerve stimulation, physiotherapy, and hydrotherapy may also be helpful. Orthopaedic devices (e.g., canes, shoe rises, etc.) may help some patients. For those patients whose pain is resistant to medical therapy, joint replacement may be indicated. Surgery may also be required in nerve compression syndromes that are nonresponsive to medical treatment. ${ }^{1}$

\section{Specific therapy}

Drug management is the mainstay of therapy for active PDB. Specific therapy is generally aimed at decreasing abnormal bone turnover due to osteoclastic bone resorption. ${ }^{1}$ Since their introduction, bisphosphonates (see Table 2) have become the gold standard of therapy for PDB. Other therapies may still be considered for patients who are intolerant of or nonresponsive to bisphosphonates. It is difficult to compare the different treatment regimens since there is a lack of consensus vis-à-vis standard therapeutic response. ${ }^{1}$ However, active head-to-head comparison trials have been conducted with many of the bisphosphonates. In general, the endpoints for these trials are SAP normalization and time to recurrence (based on rise in SAP). Results from these and ongoing trials are help- ing to provide reliable indications of comparative efficacy.

Bisphosphonates: Bisphosphonates are a class of drugs related to the naturally occurring mineralization inhibitor inorganic pyrophosphate. They are non-toxic to most tissues, as they require an acid environment to traverse cell membranes. In biological systems, they are able to bind to the surface of hydroxyapatite crystals within bone, especially on those surfaces undergoing active osteoclastic resorption, and are then able to enter the osteoclast because of the acid environment created by osteoclasts on the resorption surface. ${ }^{1}$ Bisphosphonates work according to one of two main mechanisms of action, depending on the chemical nature of the side-chain attached to the basic bisphosphonate core. For the more potent, nitrogencontaining bisphosphonates (i.e., zoledronic acid, alendronate, risedronate, and pamidronate), the direct intracellular target in osteoclasts is the enzyme farnesyl diphosphate synthase in the cholesterol biosynthetic pathway. ${ }^{23,28}$ Its inhibition suppresses a process, protein prenylation, which is essential for the basic cellular processes required for osteoclastic bone resorption and cell survival. The end result is abrupt inhibition of osteoclast activity followed by apoptosis. The relatively weak, non-nitrogen, simple bisphosphonates (i.e., clodronate and etidronate) also inhibit bone resorption through induction of osteoclast apoptosis by generating a toxic analog of adenosine triphosphate, which then targets the mitochondria, the energy centre within the cell. ${ }^{23,28}$ Clodronate is not approved for the treatment of PDB in Canada. In addition, despite its indication, both the UK guidelines and the Canadian consensus statement specifically recommend against the use of etidronate as treatment for PDB since it is not only less effective than newer bisphosphonates in suppressing biochemical markers of disease activity but, in high doses, may lead to mineralization defects.1,23

Five bisphosphonates are currently indicated in Canada for the treatment of PDB (see Table 2). These include two intravenous bisphosphonates (zoledronic acid $5 \mathrm{mg}$ and pamidronate) and three oral bisphosphonates (alendronate, risedronate and etidronate). 
TABLE 2. Specific Therapeutic Agents Indicated in Canada for the Treatment of PDB (order according to level of recommendation)

\begin{tabular}{|c|c|}
\hline I. Bisphosphonates & Administration and Dosage ${ }^{1}$ \\
\hline Zoledronic acid & -Intravenous: one single dose of $5 \mathrm{mg} / 100 \mathrm{~mL}$ given over no less that \\
\hline $5 \mathrm{mg}$ & $15 \min$ \\
\hline & -Patients must drink $\sim 500 \mathrm{~mL}$ water before and after infusion \\
\hline Trade Name: & -No restrictions on food, drink or activities \\
\hline Aclasta $^{\circledR}$ & $\begin{array}{l}\text {-Adequate intake of calcium (1000-1500 mg daily) and vitamin D } \\
(800-1000 \text { IU daily**) is recommended prior to and after bisphos- }\end{array}$ \\
\hline Manufacturer: & phonate dosing for the first two months \\
\hline
\end{tabular}

Novartis

Risedronate

$30 \mathrm{mg}$

Trade Name:

Actonel $^{\circledR}$

3 Manufacturer:

14 Procter \& Gamble

\section{Alendronate \\ $40 \mathrm{mg}$}

17

18 Trade Name:

19 Fosamax $^{\circledR}$

20 Manufacturer:

21 Merck

3 Now available as a generic

\section{Pamidronate \\ Trade Name: \\ Aredia $^{\circledR}$ \\ Manufacturer: \\ Novartis}

Now available as a generic

\section{Etidronate Disodium}

Trade Name: Didronel ${ }^{\circledR}$

Manufacturer: Procter \& Gamble 40

* Reference 1 , unless otherwise indicated. $\mathrm{BP}=$ bisphosphonate $\mathrm{SAP}=$ serum alkaline phosphatase ** The recommendation for $800-1000$ IU of vitamin D is based on expert consensus opinion (grade D recommendation)

-Tablet: $40 \mathrm{mg}$ once daily for 6 months

-Must be taken in the morning with $\sim 250 \mathrm{~mL}$ of water on an empty stomach

$\cdot 3^{\text {rd }}$ generation BP

- No food, beverages, or medications for at least 30 minutes after dose

-Patient may sit but not lie down for at least 30 minutes after taking alendronate

-Adequate intake of calcium (1000-1500 mg daily) and vitamin D (800-1000 IU daily) is recommended prior to and after bisphosphonate dosing for the first two months

-Intravenous (IV) infusion 180 to $210 \mathrm{mg}$. This may be administered either as:

-30 mg once a week for 6 weeks pearance $60 \mathrm{mg}$ as a single dose with monitoring over 1-2 months.)

-Infusions administered every 2 weeks: initial dose (week 1$)=30$ $\mathrm{mg}$; subsequent doses $($ weeks $3,5, \& 7)=60 \mathrm{mg}$; $($ total dose $=210$ $\mathrm{mg}$ )

- A course of pamidronate may be readministered at intervals as needed

-Serum creatinine should be tested before each pamidronate treatment

-Adequate intake of calcium (1000-1500 mg daily) and vitamin D (800-1000 IU daily) is recommended

-200 to $400 \mathrm{mg}$ tablet taken by mouth once daily for 6 months

-Should be taken on an empty stomach at least 2 hours before or after meals with a full glass of water

-A course of etidronate should not exceed 6 months

- Repeat courses can be given after rest periods of at least 3 months duration

-Adequate intake of calcium (1000-1500 mg daily) and vitamin D (800-1000 IU daily) is recommended
-Tablet: $30 \mathrm{mg}$ once daily for 2 months

-Must be taken in the morning with $\sim 250 \mathrm{~mL}$ of water on an empty stomach

-No food, beverages, or medications for at least 30 minutes after dose

-Patient may sit but not lie down for at least 30 minutes after taking risedronate

-Adequate intake of calcium (1000-1500 mg daily) and vitamin D

(800-1000 IU daily) is recommended prior to and after bisphosphonate dosing for the first two months

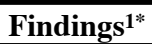

-Newest and most potent BP

- Only single-dose BP

-Most patients achieve normalization of $\mathrm{SAP}(89 \%)$ and demonstrate therapeutic response (96\%) within 6 months $^{31}$

-Superior, faster acting, longer-lasting efficacy compared to risedronate 31,32 - Can be associated with transient febrile reaction - $3^{\text {rd }}$ generation BP

-Shown to normalize SAP and improve bone turnover associated with improvement in radiological changes

-Avoids risk of inhibition of mineralization

-Demonstrated superior SAP reduction and remission $(p<0.001)$ compared to pamidronate in PDB patients previously treated with pamidronate 33

- Reduces SAP activity $50 \%$ to $80 \%$

-The recommended total dose of pamidronate for a treatment course is $\bullet$ Improves radiological and scintigraphic ap-

-Improves bone turnover (total dose $=180 \mathrm{mg}$ ). (Note that other options are used empiri- $\quad$ Reduces bone pain cally by some physicians, e.g., 3 doses of $60 \mathrm{mg}$ over $1-2$ weeks or $\bullet$ Generally well tolerated but associated with

$-1^{\text {st }}$ BP used in treatment of PDB substantial number of febrile reactions and

occasional increases in bone pain following

- Occasional phlebitis at the infusion site.
-Reduces SAP activity $40 \%$ to $70 \%$ (dose dependent)

-Improves pagetic pain

-High doses associated with GI side effects and increased risk of fracture, possibly due to focal osteomalacia which can occur within two weeks of treatment or with continuous therapy -Low-dose treatment associated with long-term resistance to treatment

-UK guidelines and Canadian Consensus do not recommend it for PDB 
When taken orally, bisphosphonates are poorly absorbed from the gastrointestinal tract and absorption is diminished when taken with food, particularly containing calcium or other divalent cations. Therefore, oral bisphosphonates must be taken with water on an empty stomach, allowing an interval of at least half an hour (and preferably more) after dose before eating or drinking. Nitrogen-containing bisphosphonates (alendronate and risedronate) can sometimes cause upper gastrointestinal side-effects, such as heartburn and dyspepsia, and a few cases of oesophageal ulceration and stricture have been reported with alendronate, due to the tablet sticking in the esophagus. Alendronate and risedronate should therefore be used with caution in patients with dysphagia, symptomatic oesophageal 6 disease, gastritis, duodenitis or ulcers, and these drugs are contraindicated when there are abnormalities of the oesophagus or other factors which delay oesophageal emptying. Patients should be instructed to stay fully upright for at least 30 minutes after oral administration. There are no restrictions with IV bisphosphonates on when and what to drink, or on normal activities such as standing, sitting, taking a walk or exercising.

Although uncommon with oral bisphosphonates, a transient, acute febrile reaction often develops after initial exposure to intravenously administered bisphosphonates. Taking acetaminophen or an NSAID can also prevent or ameliorate this acute-phase reaction. The prevalence and severity of the acute-phase reaction decreases considerably by the time of the second infusion. Patients who are prescribed an intravenously administered bisphosphonate should drink two glasses of water before and after infusion. This will ensure that renal glomerular filtration (GFR) is adequate to prevent any potential renal damage. All patients prescribed bisphosphonates should have serum calcium, serum creatinine and GFR determined prior to dosing as the agents are contraindicated if GFR is $<30$.

An adequate intake of calcium (1000-1500 mg daily) and vitamin D (800-1000 IU daily) should be ensured both before and after dosing during the first 4 two months of treatment to prevent post-dose hypocalcaemia.
Osteonecrosis of the jaw $(\mathrm{ONJ})$ is a rare condition of unknown aetiology that most often occurs after the treatment of cancer patients, receiving high-dose, frequent intravenous bisphosphonate injection or infusion. Since 2003, there have been a few case reports of ONJ after oral bisphosphonate treatment for osteoporosis. This has required a class-effect warning for all bisphosphonates. Patients present, usually after dental trauma or surgery, with areas of exposed mandibular or maxillary bone that fails to heal after at least six weeks of conservative therapy. No cases of ONJ have been reported in PDB patients treated with zoledronic acid $5 \mathrm{mg}$. Nonetheless, a dental examination with appropriate preventive dental care should be recommended for patients with dental risk factors. Since the concern about ONJ is increasing and there are still a paucity of data, the ASBMR and other societies and organizations have promulgated various recommendations/guidelines. The task force of the ASBMR published recommendations in 2006, which should serve as guideline advice for practitioners until more information and a reliable ONJ incidence is determined. ${ }^{29}$

Normalization of biochemical markers (e.g., SAP) implies disease remission and is now the goal of therapy. Newer bisphosphonates (i.e., alendronate, risedronate and zoledronic acid $5 \mathrm{mg}$ ) have made normalization of biochemical indices achievable.

Although bisphosphonates have a qualitatively similar effect on bone resorption, their distinct physicochemical and biologic properties prevent reliable extrapolation of therapeutic efficacy from one agent to another. ${ }^{23}$ Studies have assessed whether resistance to one bisphosphonate would predict resistance to others and found that failure to achieve biochemical normalization was likely to be drug-specific and not necessarily indicative of a general inability to respond to bisphosphonate therapy. ${ }^{30}$

Comparative trials have been published evaluating the relative efficacy of the bisphosphonates in the treatment of PDB. ${ }^{31-33}$ These trials typically use extent of suppression of SAP and duration of remission as evidence of superior treatment effect. Although of somewhat differing protocols, these trials demonstrate 44 that alendronate and risedronate are superior to eti- 45 
dronate, and risedronate appears superior to alendronate. Recent comparison of zoledronic acid $5 \mathrm{mg}$ and risedronate in 357 patients after six months showed normalization of SAP in $89 \%$ of zoledronic acid-treated patients and $58 \%$ of risedronate-treated patients. ${ }^{31}$ Patients were given either zoledronic acid (one $5 \mathrm{mg}$ infusion) or oral risedronate $(30 \mathrm{mg}$ daily for two months) and evaluated six months after commencing therapy. Patients in remission at that point were followed for duration of response and, after 18 1 months, zoledronic acid $5 \mathrm{mg}$ extended remission in $98 \%$ of patients with one single dose, compared to $66 \%$ with risedronate. ${ }^{32}$ Subsequent analyses have documented differences in cost-effectiveness in favour of the more effective therapies. ${ }^{34,35}$

16

17 Surgery: In treating PDB, surgery is generally con18 fined to the management of fracture, deformity, or ar19 thritis. There may be an increased risk of blood loss 20 during surgery since pagetic bone is more vascular than healthy bone. ${ }^{1}$ Therefore, the administration of bisphosphonate therapy prior to surgery is recommended, "if only to ensure that treatment of the underlying disease has not removed the need for surgery."1 Bisphosphonate therapy may also be important in optimizing bone strength and quality prior to surgery to enhance surgical outcomes. Surgery may also be recommended for patients with painful pagetic deformities and it is also suggested for patients with osteoarthritis related to PDB whose symptoms do not improve with medical therapy. ${ }^{1}$

\section{Follow-up}

The goal in treating PDB is to relieve symptoms and prevent complications. In addition to patient reports of pain relief and radiological improvement of osteolytic lesions, it is usual to assess treatment efficacy by measuring biochemical markers of bone turnover. Optimum levels of bone turnover reduction have not been established, but the consensus is that biochemical markers should be suppressed into the population reference (normal) range. ${ }^{1} \mathrm{SAP}$ is the most commonly used biochemical marker because it has high reproducibility and is sensitive to clinically important
TABLE 3. Follow-up and Treatment Endpoints

SAP

- Repeat at 6 and 12 months, then annually or in the event of new symptoms

- Normalization is the goal (re-treat after minimum of 6 months)

- Increase in SAP of $25 \%$ above nadir or SAP above ULN

If SAP is normal:

- Re-treat after minimum of 6-12 months if there is persistent abnormal uptake on bone scan consistent with PDB

- Re-treat after minimum of 6-12 months if there are persistent osteolytic lesions on xray of a known pagetic site (especially the weight-bearing long bones).

changes in disease activity. ${ }^{1}$ It is also widely available and relatively inexpensive. SAP is elevated in untreated PDB (correlated with disease extent) and usually falls within seven to 10 days of initiating antiresorptive therapy. A decrease of $25 \%$ in SAP represents a significant response. ${ }^{1}$ Because in all studies of bisphosphonate therapy the nadir SAP level occurred three to six months after initiation of therapy and was followed by a gradual offset of treatment effect, it is recommended that bone turnover be measured after six months of therapy, and thereafter at intervals of one year (see Table 3 ). ${ }^{1}$ Although bone specific ALP is more sensitive and specific, the difference is unlikely to be of clinical importance except for patients with liver disease, patients with monostotic disease, and those with total SAP within the normal range. ${ }^{1}$

Urinary markers of bone resorption such as deoxypyridinoline, alpha-C-telopeptide or hydroxyproline respond more quickly to treatment (nadir within 10 to 30 days post treatment) and may also indicate relapse before changes in SAP occur. However, they are not recommended (nor, for the most part, necessary) since their reproducibility is lower than that of SAP and changes greater than $40 \%$ are often required at the individual level to be significant. ${ }^{1}$

Isotope bone scans are a relatively insensitive means of measuring response and there is a considerable delay between biochemical response and improvement in bone scans. Moreover, they expose the patient to extra radiation. Nevertheless, they may be 
TABLE 4. Recommendations for Treatment of Active PDB

First choice: zoledronic acid $5 \mathrm{mg}$ IV

Second choice: risedronate or alendronate oral

Third choice: pamidronate IV

Other choices (indicated but not recommended): etidronate, calcitonin

\section{Choice of treatment depends on:}

- Efficacy

- \% of patients responding to therapy

- Duration of remission

- Adherence

- Convenience

- Tolerability

- Cost

5 useful in monostotic disease and in situations in which 16 pain persists despite normal bone biochemistry. ${ }^{1}$ 17

\section{Treating relapse}

The reintroduction of bisphosphonate therapy is indicated for patients who relapse. The parameters for retreatment are as follows: ${ }^{1}$ Symptom relapse or persistence, usually pain, should be confirmed by objective evidence of disease activity. In its absence, other causes of pain should be sought.

Biochemical criteria must be used to measure therapeutic efficacy in asymptomatic disease. It is generally accepted that an increase of SAP of $25 \%$ above nadir, even if the total is still within the normal range, indicates significant relapse. ${ }^{1}$

As stated above, the effects of bisphosphonate treatment are generally apparent within three months and maximal by six months. Thus, it is appropriate to review and change treatment if a patient has failed to respond in six months. It is our position that high potency bisphosphonates should be used to initiate treatment (see Table 4).

\section{Pharmacoeconomics: the cost of treatment}

40 In the treatment of $\mathrm{PDB}$, a more complete remission 41 with a longer duration induced by a more effective 42 albeit more expensive treatment is likely to be more 43 cost-effective. Such analyses have been undertaken 44 for etidronate, alendronate and risedronate 23 based 45 upon the head-to-head trials available. When zole- dronic acid $5 \mathrm{mg}$ therapy is considered in this context, its cost-effectiveness surpasses that of previously evaluated bisphosphonates.

In a Dutch study of published trials, the relative cost-effectiveness of zoledronic acid $5 \mathrm{mg}$ and risedronate, including all direct medical and travel costs, were modeled over 24 months. In the zoledronic acid group, 19.6 months were spent with normalized SAP levels, versus 13.2 for the risedronate group. The number of treatments per year was 0.58 for the zoledronic acid group and 0.94 for the risedronate group. Management costs in the zoledronic acid group were lower ( $€ 891 ; \sim \$ 1,222 \mathrm{CAN}$ ) than in the risedronate group ( $€ 1217 ; \sim \$ 1,675 \mathrm{CAN}$ ), leading to savings of $€ 326$ ( $\sim 449 \mathrm{CAN})$ per year. The authors concluded that treatment of PDB with zoledronic acid $5 \mathrm{mg}$ is more effective and less expensive than treatment with risedronate. ${ }^{34}$

A recently presented economic evaluation of the treatment of PDB, using data from the Canadian provincial healthcare system, has also shown that zoledronic acid $5 \mathrm{mg}$ is clinically and economically superior over two years to risedronate. ${ }^{35}$ The target population was PDB patients with SAP levels at least twice the upper limit of normal, symptomatic, or at risk of PDB complications. The treatment cost was determined using efficacy and safety data obtained from a pooled analysis of two recently published comparative trials. The direct medical costs included drug, physician, remission, laboratory, diagnostic and adverse event costs (see Table 5 for a breakdown of costs). The frequency of physician visits, laboratory and diagnostic tests were based on published treatment guidelines (no infusion costs for zoledronic acid $5 \mathrm{mg}$ were included since the manufacturer would fund the infusions). A 5\% discount rate was used after one year. The acquisition cost of zoledronic acid $5 \mathrm{mg}$ was equivalent to that of risedronate.

A single 5-mg IV dose of zoledronic acid $5 \mathrm{mg}$ was shown clinically to have superior efficacy, faster onset and longer-lasting effect compared to oral risedronate $30 \mathrm{mg}$ daily for 60 days. ${ }^{35}$ Overall PDB treatment costs were $\$ 2,057.66$ for zoledronic acid $5 \mathrm{mg}$ and $\$ 2,542.16$ for risedronate (\$CAN, 2005). ${ }^{35} \mathrm{~A}$ sensitivity analysis demonstrated that a $2.7 \%$ increase in 
TABLE 5. Cost Breakdown* of PDB Treatment over 2 Years ${ }^{35}$

\begin{tabular}{|c|c|c|}
\hline & Zoledronic acid $5 \mathrm{mg}$ & Risedronate $30 \mathrm{mg}$ \\
\hline Initial drug cost & $\$ 716.04$ & $\$ 716.04$ \\
\hline $\begin{array}{l}\text { Drug cost due to } \\
\text { relapse }\end{array}$ & $\$ 157.21$ & $\$ 600.44$ \\
\hline $\begin{array}{l}\text { Additional drug cost } \\
\text { (antipyretic and } \\
\text { opiate agonist) }\end{array}$ & $\$ 679.42$ & $\$ 679.42$ \\
\hline Infusion cost & Supported by Novartis & None \\
\hline Physician cost & $\$ 404.80$ & $\$ 404.80$ \\
\hline Laboratory cost & $\$ 78.33$ & $\$ 110.33$ \\
\hline Diagnostic cost & $\$ 19.89$ & $\$ 29.98$ \\
\hline $\begin{array}{l}\text { Adverse event cost } \\
\text { (flu-like symptoms) }\end{array}$ & $\$ 1.97$ & $\$ 1.15$ \\
\hline Total treatment cost & $\$ 2057.66$ & $\$ 2542.16$ \\
\hline Cost difference & & $+\$ 484.50$ \\
\hline
\end{tabular}

* \$CAN, 2005

the price of risedronate resulted in a $7.3 \%$ increase in the cost savings for zoledronic acid $5 \mathrm{mg}$, from $\$ 484.50$ to $\$ 519.69$. The higher remission rates, faster onset and longer-lasting effect of zoledronic acid $5 \mathrm{mg}$ compared with risedronate resulted in drug cost savings of $\$ 443.23$. No incremental cost-effectiveness ratio was calculated as treatment with zoledronic acid $5 \mathrm{mg}$ was clinically more effective and cost less than risedronate. ${ }^{35}$

\section{Conclusion}

Paget's disease of bone is characterized by accelerated bone resorption followed by the deposition of dense and disorganized bone matrix. The disease may be asymptomatic or symptomatic, depending on the 4 bones involved. The most common symptom is pain in the affected bone. Normally affecting the elderly, 36 the overall incidence of PDB in Caucasians is estimated to be $\sim 3 \%$. The incidence appears to be decreasing. In general, diagnosis may be confirmed both by X-ray and by the biochemical marker SAP, 40 which is elevated in $85 \%$ of individuals with untreated 41 PDB. Treatment of PDB is indicated for all patients 2 with symptoms as well as for those asymptomatic 3 patients with active PDB in areas of the skeleton with 44 the potential to produce complications of clinical sig- nificance. The recommendation is to treat with bisphosphonates that have demonstrated superior efficacy and remission rates.

Methodology: No specific process was used for the development of this position paper. However, a formal literature search was carried out using standard search engines (for example, PubMed). We also relied heavily on the bibliography from the recent UK guidelines.

Conception and Acknowledgements: This paper came together as a result of a meeting between the authors and Novartis Canada, Inc. that took place to review data from various clinical trials the authors were involved in. It was proposed by the academic faculty that we create Paget's disease treatment recommendations with a Canadian perspective using the UK guidelines as a template but representing Canadian recommendations. The production of this position paper was supported by an unrestricted grant from Novartis Canada, Inc., who had no part in the content of the paper. None of the authors have received or will receive any compensation or remuneration in any form for their contribution to this manuscript.

The paper has been endorsed by the Canadian Society of Endocrinology and Metabolism. Four of the authors RGJ, DAH, DK and LGSM are all members of CSE. However, the paper was independently reviewed by two other members of CSEM, who are experts in metabolic bone disease.

\section{References}

1. Selby PL, Davie MWJ, Ralston H, et al. Guidelines on the management of Paget's disease of bone. Bone 2002;31:10-9.

2. Cooper C, Harvey NC, Dennison EM, and van Staa TP. Update on the Epidemiology of Paget's Disease of Bone. J Bone Miner Res 2006;21:P3-P8.

3. Boland MJ, Tong PC, Naot D, Callon KE, Wattie DJ, Gamble GD, and Cundy T. Delayed Development of Paget's Disease in Offspring Inheriting SQSTM1 Mutations. J Bone Miner Res 2007;22:411-5.

4. Altman RD. Epidemiology of Paget's disease of bone. Clin Rev Bone Miner Metab 2002;1:99-102. 
5. Reddy SV. Aetiology of Paget's disease and osteoclast abnormalities. J Cell Biochem 2004;93:688-96.

6. Selby PL, Davies M, and Mee AP. Canine Distemper Virus Induces Human Osteoclastogenesis Through NF- $x$ B and Sequestosome 1/P62 Activation. J Bone Miner Res 2006;21:1750-6.

7. Roodman GD, Windle JJ. Paget's disease of bone. J Clin Invest 2005;115:200-8.

8. Ralston SH. Pathogenesis of Paget's disease of bone. Clin Rev Bone Miner Metab 2002;1:109-14.

9. Singer FR, Gruber HE, Windle JJ, Roodman GD. Ultrastructure of bone cells in Paget's disease of bone. J Bone Miner Res 2006;21(Suppl 2):P51-P54.

10. Siris ES, Ottman R, Flaster E, Kelsey JL. Familial aggregation of Paget's disease of bone. J Bone Miner Res 1991;6:495-500.

11. Morales-Piga AA, Rey-Rey JS, Corres-Gonzalez J, Garcia-Sagredo JM, Lopez-Abente G. Frequency and characteristics of familial aggregation of Paget's disease of bone. J Bone Miner Res 1995;10:663-70.

12. Fotino M, Haymovits A, Falk CT. Evidence for linkage between HLA and Paget's disease. Transplant Proc 1977;9:1867-8.

13. Tilyard MW, Gardner RJ, Milligan L, Cleary TA, Stewart RD. A probable linkage between familial Paget's disease and the HLA loci. Aust N Z J Med 1982;12:498-500.

14. Cody JD, Singer FR, Roodman GD, et al. Genetic linkage of Paget disease of the bone to chromosome 18q. Am J Hum Genet 1997;61:1117-22.

15. Laurin N, Brown JP, Lemainque A, et al. Paget disease of bone: mapping of two loci at 5q35-qter and 5q31. Am J Hum Genet 2001;69:528-43.

16. Hocking LJ, Herbert CA, Nicholls RK, et al. 2001 Genomewide search in familial Paget disease of bone shows evidence of genetic heterogeneity with candidate loci on chromosomes 2q36, 10p13, and 5q35. Am J Hum Genet 2001;69:1055-61.

17. Good DA, Busfield F, Fletcher BH, et al. Linkage of Paget disease of bone to a novel region on human chromosome 18q23. Am J Hum Genet 2002;70:517-25.

18. Laurin N, Brown JP, Morissette J, Raymond V. Recurrent mutation of the gene encoding sequestosome 1 (SQSTM1/p62) in Paget disease of bone. Am J Hum Genet 2002;70:1582-8.

19. Morissette J, Laurin N, Brown JP. Sequestosome 1: Mutation Frequencies, Haplotypes, and Phenotypes in
Familial Paget's Disease of Bone. J Bone Miner Res 2006;21:P38-P44.

20.Layfield R, Hocking LJ. SQSTM1 and Paget's disease of bone. Calcif Tissue Int 2004;75:347-57.

21.Kanis JA. Pathophysiology and treatment of Paget's disease of bone. 2nd edition London:Martin

Dunitz:110-38,1998.

22. Devogelaer J-P. Modern therapy for Paget's disease of bone. Focus on bisphosphonates. Treat Endocrinol 2002;1:241-57.

23. Drake WM, Kendler DL, Brown JP. Consensus statement on the modern therapy of Paget's disease of bone from a Western Osteoporosis Alliance Symposium. Clin Ther 2001;23(:620-6.

24. Alexandersen P, Peris P, Guanabens N, et al. Nonisomerized c-telopeptide fragments are highly sensitive markers for monitoring disease activity and treatment efficacy in Paget's disease of bone. J Bone Miner Res 2005;20:588-95.

25. Morris CD, Einhorn TA. Bisphosphonates in orthopedic surgery. J Bone Joint Surg Am 2005;87:1609-18.

26. Melton III LJ, Tiegs RD, Atkinson EJ, O'Fallon WM. Fracture risk among patients with Paget's Disease: A population-based cohort study. J Bone Miner Res 2000;15:2123-8. Abstract available at www.jbmronline.org/doi/abs/10.1359/jbmr.2000.15.11 .2123 .

27. Meunier PJ, Vignon E. Therapeutic strategy in Paget's disease of bone. Bone 1999;17(5 suppl):489S-491S.

28.28. Reszka AA, Rodan GA. Mechanism of action of bisphosphonates. Curr Osteo Reports 2003;1:45-52.

29. Shane E, Goldring S, Christakos S et al. Osteonecrosis of the Jaw: More Research Needed. J Bone Miner Res 2006;21:1503-5.

30.Papapoulos SE, Eekhoff EMW, Zwinderman AH. Acquired resistance to bisphosphonates in Paget's disease of bone. J Bone Miner Res 2006;21(suppl 2):88-91.

31. Reid IR, Miller P, Lyles K et al. Comparison of a single infusion of zoledronic acid with risedronate for Paget's disease. N Engl J Med 2005;353:898-908.

32. Hosking D, Lyles K, Brown JP et al. Long-Term Control of Bone Turnover in Paget's Disease with Zoledronic Acid and Risedronate. J Bone Miner Res 2007;22:142-8.

33. Walsh JP, Ward LC, Stewart GO, et al. A randomized clinical trial comparing oral alendronate and intrave- 
nous pamidronate for the treatment of Paget's disease of bone. Bone 2004;34:747-54.

34. Al M, Groot M, Galani C, Engbersen A. Economic evaluation of long term management strategies for Paget's disease of bone. Abstract SA465. ASBMR 27th Annual Meeting, Nashville, Tennessee 2005.

35. Mittman N, Isogai PK, Adachi JD, Kindunu CM, Barbeau M. Economic evaluation of Aclasta ${ }^{\circledR}$ (zoledronic acid $5 \mathrm{mg}$ ) versus Actonel ${ }^{\circledR}$ in treatment of Paget's disease of bone. A Canadian perspective. Poster presentation, March 7, 2006; ECCE 2006, Vienna.

16 Dr. Robert G. Josse, Director, Osteoporosis Centre,

17 St. Michael's Hospital

1861 Queen Street East,

19 6th Floor, Suite 6122,

19 Toronto, Ontario M5C 2T2

josser@smh.toronto.on.ca 